\title{
Ein Fall von Zwergwuchs und Idiotie nebst Bemerkungen über die Klassifikation der Zwerge.
}

Von

Dr. W. M. van der Scheer, Meerenberg (Holland).

Mit 4 Textfiguren und 4 Tafeln.

(Eingegangen am 31. Juli 1915.)

\section{Essentieller Zwergwuchs.}

Der Name Zwerg soll nur ausdrücken, daß das betreffende Individuum in Vergleich zu anderen Individuen desselben Alters, desselben Geschlechts und derselben Rasse eine erhebliche Reduktion seiner Körpergröße aufweist. Diese Reduktion der Körpergröße hat meistens nur die Bedeutung eines Symptoms, aber es sind einige Fälle bekannt, wo sie das einzige Unterscheidungsmerkmal des Individuums seinen Rasseund Geschlechtsgenossen gegenüber bildete.

Hastings-Gilford spricht dann von essentiellem oder unkompliziertem Zwergwuchs $s^{1}$ ).

Man findet in diesen Fällen eine harmonische Reduktion des ganzen Körpers.

Außer diesen quantitativen Unterschieden sind diese Menschlein als vollkommen normal zu betrachten. Intelligenz, Lebensfähigkeit, Widerstandsvermögen, geschlechtliche Entwicklung usw. sind in jeder Hinsicht dieselben wie die aller Individuen ihrer Rasse. Es liegt auf der Hand, daß hier die Grenze zwischen normal und abnormal sehr schwer zu ziehen ist. Die essentiellen Zwarge zeigen unter sich keine Familienähnlichkeit. Heredität ist nicht nachzuweisen.

\section{Ateleiosis.}

Bei weitaus der Mehrzahl der vorkommenden und beschriebenen Zwerge gibt es außer den quantitativen Untersci ieden andere Erscheinungen, die das Individuum als von der Norm abweichend kennzeichnen, so daß in diesen Fällen der Name des essentiellen oder unkomplizierten $\mathrm{Z}$ wergwuchses nicht angewendet werden darf.

1) Levi nennt diese Fälle essentielle Mikrosomie und definiert diese genau wie Hastings - Gilford seinen essentiellen Zwergwuchs. Nur passen die Fälle Levis für den größten Teil gar nicht zu seiner Definition und müssen zu einer ganz anderen Gruppe von Zwergen gercehnet werden (s. später).

z. f. d. g. Neur. u. Psych. O. XXXII. 
Hierzu gehören u. a. auch die Fälle, die Falta ,echte Zwerge“" nennt, als deren Charakteristicum er das Wohlproportioniertsein des Individuums betrachtet. Er faßt sie aber nicht als einheitliche Krankheit auf und unterscheidet in großen Zügen zwei Typen:

A. die primordiale Nanosomie,

B. den Palta ufsohen Zwerg.

Der primordiale $Z_{w e r g}$ wird als proportionierter $Z_{w e r g}$ dadurch charakterisiert, daß der Zwergwuchs von Geburt an besteht, daß aber die weitere Entwicklung, abgesehen von der Reduktion der Körpergröße in der normalen Weise stattfindet. Die Genitalentwicklung, die Verknöcherung der Epiphysärscheiben, die Intelligenz sind nahezu normal zu nennen.

Der Paltaufsche Zwerg, die infantile Form Hansemans, wird am reinsten durch den von Palta uf beschriebenen $Z$ werg repräsentiert. Das Individuum hat als Neugeborener normales Aussehen und zeigt anfangs eine normale Entwicklung. Erst später, wenn auch schon sehr jung, tritt plötzlich ein Stillstand oder besser, ein erhebliches Zurückbleiben des Wachstums auf.

Die Epiphysenscheiben schließen sich nicht. Die primären und sekundären Geschlechtskennzeichen bleiben in ihrer Entwicklung zurück.

Was den primordialen Zwerg Hansemans anbelangt, so müßte diese Form nach der Benennung vollkommen in das Bild des schon genannten essentiellen $Z$ wergwuchses passen, wo nur der quantitative Unterschied den Zwergwuchs charakterisiert, wo wir also den echten diminutiven Menschen, das von Levi als ,,mikrosom" scharf definierte Individuum haben würden.

Untersuchen wir jedoch die Fälle der Literatur, dann stellt sich heraus, daß bei weitaus der Mehrzahl der als echte Zwerge beschriebenen Fälle die obige Definition nicht anwendbar ist.

Wenn Falta sagt, daß das Genitale, die Verknöcherung der Epiphysenfugen und die Intelligenz sich ziemlich normal (von mir gesperrt) entwickelt, dann spricht er dadurch mehr aus als einen quantitativen Unterschied dem Normalen gegenüber.

Sehr häufig findet man weiter infantile Züge (H a nse mans eigener Fall) und tatsächlich gibt es zwischen diesen zwei künstlich geschiedenen und in ihren äußersten Repräsentanten auch sehr verschiedenen Formen, so zahlreiche Utbergänge, daß sehr viele der Fälle unmöglich unter einen der aufgestellten Typen unterzubringen sind (z. B. die Fälle Joachimsthals und Kraus').

Der Name ,echter Zwergwuchs" scheint mir deshalb für diese Fälle kein glücklich gewählter.

Außer dem Zwergwuchse können so viele andere Erscheinungen 
vorkommen, wie z. B. rudimentäre Genitalia, Spätentwicklung der sexuellen Eigenschaften, offene Epiphysenscheiben, daß das klinische Bild sicher nicht ausschließlich durch quantitative Verhältnisse bestimmt wird, sondern auch qualitativen Veränderungen große Bedeutung zukommt. Der als Charakteristicum angegebene Unterschied des Wohlproportionierten gilt für die meisten dieser Fälle auch sicher nicht.

Proportioniert würden wir ein Individuum nennen, wenn die Körperverhältnisse übereinstimmen mit denen eines gleichalterigen normalen Individuums. Jedes Alter hat seine eigenen Proportionen. Das Verhältnis zwischen den verschiedenen Körperteilen ist bei Kindern vollkommen anders wie bei Erwachsenen. Arme und Beine sind im Verhältnis zum Rumpf beim Kind so viel kürzer wie beim Erwachsenen, daß es nicht berechtigt erscheint, einen $Z$ werg, der z. B. 30 Jahre alt, die Proportionen eines fünfjährigen Kindes zeigt, und also im Verhältnis zum Rumpf einen viel zu großen Kopf und viel zu kurze Extremitäten hat, proportioniert zu nennen; und weitaus die Mehrzahl der beschriebenen ,echten $Z_{w e r g e "}$ " zeigen einen relativ zu großen Kopf und relativ zu kurze Arme und Beine.

Wohl besteht in den meisten dieser Fälle ein normales Verhältnis zwischen Weichteilen und Skelett, und dies ist auch wohl der Grund, daß man diese Formen als proportionierte Zwerge von einer Gruppe anderer Wachstumsstörungen abgegrenzt hat, wo eine unregelmäßige auf das Skelett beschränkte Störung, die die Weichteile nicht befällt, eine Disproportion hervorruft - auch zwischen Skelett und Weichteilen.

Weder der Name des ,echten“, noch das Kennzeichen des „proportionierten" ist also richtig.

Dennoch gibt es Hinweise genug, die es rechtfertigen, solange ätiologische und pathogenetische Momente vollkommen fehlen, diese Zwerge vom klinischen Standpunkte aus in einer Gruppe unterzubringen. Es ist das Verdienst Hastings-Gilfords, die bestimmten Charakteristica dieser Gruppe scharf betont zu haben.

Er weist erstens darauf hin, daß in allen typischen Fällen eine Familienähnlichkeit besteht, ebenso wie bei Mongolen und Kretinen. Sehr schön zeigt dies eine Tafel aus Gilfords großzügiger Arbeit (S. 586), wo er nebeneinander 20 Bilder dieser Art Zwerge reproduziert. Der relativ zu große Kopf, das breite flache Gesicht, wie man es im Kindesalter trifft, bevor noch Nase und Oberkiefer angewachsen sind, und scharfe mehr ausgesprochene Züge hervorgerufen haben, sind auffällig.

„The features are those of stereotyped childhood. Hence the stature is small, the limbs short, the head large, and the face broad and flat, the bridge of the nose is undevelopped and the distance from the ear to the vertex is comparatively great. The facial type is so well defined 
in some cases as to obliterate the natural expression of character and produce a strong resemblance between dwarfs of different families. But added to these childish features are the lines and superficial marked of age."

Als zweite charakteristische Eigenschaft nennt Gilford das Fehlen irgendeines nachweisbaren schädlichen Einflusses.

Oft werden die Kinder zu klein geboren. Oft bleibt das Wachstum zurück, eine Ursache ist aber nicht zu eruieren.

Als drittes Charakteristicum nennt er die deutlich ausgesprochene Heredität. Er nennt diese Entwicklungsstörung Ateleiosis und unterscheidet:

1. die sexuelle Varietät, wo in der Pubertät die Genitalia sich entwickeln, die Epiphysärscheiben sich schließen und jedes weitere Wachstum aufhört. Diese Form hat also die meiste Ähnlichkeit mit dem primordialen Zwergwuchs mit kindlichen Proportionen;

2. die asexuelle Varietät, wo die Epiphysärscheiben offen bleiben und sich die Genitalia und sekundären Geschlechtsmerkmale nur in geringem Maße entwickeln, eine Beschreibung, die vollkommen stimmt zu dem von Hansemann als infantilen, von Falta als Palta ufschem Zwerg bezeichnetem Typ.

Zwischen beiden gibt es zahlreiche UUbergänge (Fälle von Joa chims thal), was gewiß auf die Verwandtschaft hinweist.

Bei der ersten Form scheint ein verspätetes Auftreten der Geschlechtsreife nicht selten zu sein.

Noch schärfer tritt der engere Zusammenhang zwischen diesen zwei Formen hervor, wenn man weiß, daß die sexuelle Varietät der Ateleiosis Kinder hat, wovon einige zu dem asexuellen Typ gehören.

Gilford bespricht einen Großvater, $120 \mathrm{~cm}$ groß, mit zwei Kindern der sexuellen Varietät der Ateleiosis und reproduziert sein Bild: das eine dieser zwei Kinder hat $u$. a. ein Kind, das ein asexueller ateleiotischer Zwerg ist.

Nach dieser Beschreibung braucht es keines Beweises mehr, daß die Fälle Levis zu dieser interessanten Gruppe gehören.

Wenn wir in seinem Falle I den großen Kopf mit der Einsenkung der Nasenwurzel, worauf Levi selbst hinweist, sehen, die kurzen Extremitäten, die nach den mitgeteilten Maßen viel zu kurz sind, daneben den zwölfjährigen Sohn mit einer somatischen Entwicklung, die übereinstimmt mit der eines zwei- bis vierjährigen Kindes, a uch was die Körperproportionen betrifft, und die nach Levi als infantil aufgefaßt werden muß, sehen wir eine vollkommene Ubereinstimmung mit der sexuellen und asexuellen Varietät von Gilfords Ateleiosis. Wie schön die verschiedenen Auseinandersetzungen und die genauen Beschreibungen Levis auch sind, so verstehe ich doch nicht, wie er in 
seinem ersten Falle mit dergleichen Proportionen von ,Microsomie pure" sprechen kann, ihn also als einen Miniaturmenschen auffaßt, sogar nachdem er selbst auf die Disproportion zwischen Kopf, Arm und Beinen hingewiesen hat.

Wenn dann noch der Sohn beschrieben wird, und er sagt: ,Au syndrôme microsomie pure se sont ajoutés quelques symptômes surs d'infantilisme" ${ }^{6}$, verliert die Beobachtung nicht, wohl aber die von Levi aufgestellte Klassifikation ihren Wert.

\section{Symptomatischer Zwergwuchs.}

Es bleiben noch eine große Zahl Fälle übrig, die weder zu den essentiellen, noch zu den ateleiotischen Zwergen gehören und wo man den Zwergwuchs als Symptom aufzufassen hat.

In manchen Fällen ist uns die Ätiologie, in anderen die Pathogenese bekannt, in noch anderen tasten wir vollkommen im Dunkeln.

A. Als spezielle Gruppe dürfen wir die Fälle betrachten, wo der Zwergwuchs Folge ist einer sich hauptsächlich im Skelett abspielenden krankhaften Störung, wie z. B. die rachitischen, osteomalacischen, tuberkulösen und achondroplastischen Skelettdeformitäten.

Daß es hier aber noch fraglich ist, ob man es ausschließlich mit einer Skelettkrankheit zu tun hat, dafür brauche ich nur auf die Rachitis und die Achondroplasie hinzuweisen.

Wie dem auch sei, jedenfalls ist eine der obengenannten wohl gekennzeichneten Krankheiten als Ursache des Zwergwuchses zu betrachten.

B. In den meisten Fällen aber ist der Zwergwuchs aufzufassen als der A usdruckeiner allgemeinen Entwicklungsstörung, welche nicht nur das Skelett trifft, sondern auch die anderen Organe, das eine mehr, das andere weniger.

In einigen Fällen ist nun die Ätiologie dieser Entwicklungsstörung teilweise bekannt.

Ausfall der Funktion einiger Blutdrüsen, Schilddrüse, Thymus, Hypophysis, Nebennierenrinde, Pankreas führen zu Ernährungsstörungen, die mit ausgesprochenem Zwergwuchs kombiniert sein können, so daß man von myxödematösem, hypophysärem, glandulärem Zwergwuchs gesprochen hat. Schlechte äußere Umstände, ernste Erkrankungen in der Jugend, toxische Einflüsse, intestinale Störungen, Leber- und Milzerkrankungen (Berliner klin. Wochenschr. 35, 997, 1898) können Stillstand oder Zurückbleiben des Wachstums und der Entwicklung hervorrufen. Viele dieser genannten Ursachen werden zu dem von Falta als klinische Einheit aufgefaßten Bild des reinen Infantilismus führen. Er definiert dieses als ,ein Stehenbleiben des ganzen Organismus auf kindlicher 
Entwicklungsstufe. Das Blutdrüsensystem bleibt ebenso kindlich wie das Skelett oder der hämatopoetische Apparat oder das Zentralnervensystem, es ist daher die Entwicklungshemmung des Blutdrüsensystems der des ganzen Organismus koordiniert".

In der Mehrzahl dieser letzten Fälle ist die Wachstumstörung nicht sehr stark, und der Minderwuchs ist die Regel, doch gibt es auch Zwerge, deren Entwicklung auf eine der obengenannten Ursachen zurückgeführt werden kann.

Bei allen diesen Formen des $Z$ wergwuchses läßt sich im Gegensatz zu den essentiellen und ateleiotischen $Z$ wergen ein schädlicher Einfluß nachweisen.

Diese Individuen zeigen irgendein Zeichen einer Erkrankung oder Schwäche oder jedenfalls datiert die Entwicklungsstörung von einer Erkrankung oder einem Vorkommnis, dem offensichtlich eine große Bedeutung im Leben des Individuums zukommt.

Bei jedem Zwerg wird man also danach streben müssen, eine Ursache des Zwergwuchses nachzuweisen.

Auch bei einigen Formen der Idiotie, bei Hirnherden, bei Hydrocephalus sind Fälle von Zwergwuchs beschrieben, wo uns der direkte Zusammenhang der Erscheinungen entgeht.

In einem auf dem internationalen Kongreß für Neurologie und Psychiatrie im August 1913 gehaltenen Vortrag ist durch Professor W. We ygandt aufs neue die Aufmerksamkeit auf die Fälle von Zwergund Minderwuchs bei jugendlichen Geistesschwachen gerichtet worden.

Er bespricht die verschiedenen Formen von Minderwuchs mit ihrer Ätiologie und erörtert, in wieweit bei jeder Gruppe die Psyche und das Zentralnervensystem betroffen ist.

Er weist besonders auf die Bedeutung der Hypophysis hin; nicht nur bei den Fällen mit Hypopituitarismus, sondern auch in den Fällen von Hydrocephalie mit Zwergwuchs muß an den Einfluß des Hydrocephalus durch das Infundibulum auf die Hypophyse gedacht werden, auch in den Fällen von encephalitischer Mikrocephalie mit Minderwuchs und Zwergwuchs ist wohl daran zu denken, ob der entzündliche Prozeß nicht auch die Hypophysis in Mitleidenschaft gezogen hat. Auch in den Fällen von Encephalitis in der Jugend kann nach Weygandt an eine durch den Krankheitsprozeß bedingte Affektion der Hypophysis gedacht werden.

Im selben Zusammenhang will er die von Bourne ville aufgestellte. Form des „Nanisme diplégique" auffassen.

Er sagt zum Schluß:

„Aus der ganzen Ubbersicht ergibt sich, wie ungemein mannigfach 
die Ursachen sein können, aus denen Minderwuchs mit Schwachsinn und Hirnkrankheit hervorgehen. Angesichts der häufigen Kombination körperlicher mit psychischer Hemmung ist es dringend erforderlich, jedem der verschiedenen Symptome volle Aufmerksamkeit zu schenken, und auch schon zwecks Verhütung einer weiteren Degeneration der menschlichen Rasse, den Fällen von Minderwuchs, wegen der häufigen Verbindung mit Schwachsinn, lebhaftes Interesse zu widmen."

Es möge die Mitteilung eines Falles von Idiotie mit Zwergwuchs folgen, der in der Deutung der verschiedenen Erscheinungen zahlreiche Schwierigkeiten bietet und einzelne ätiologische und pathogenetische Fragen in den Vordergrund rückt.

S. van W., 32 Jahre alt, wurde am 5. August 1912 in Meerenberg aufgenommen. Vor dieser Zeit war sie Patientin im „Wilhelmina Gasthuis" in Amsterdam.

Aus den Nachrichten, welche ich von der Mutter und einer älteren Schwester bekam, entnehme ich folgendes:

Der Vater starb an einer Bruchoperation. Die Mutter ist gesund. Beide waren nervös.

Patientin ist das sechste Kind. Ihr folgen noch zwei; keine Fehlgeburten. Alle Kinder sind gesund mit Ausnahme unserer Patientin.

Bei ihrer Geburt wurde nichts Außerordentliches an ihr bemerkt; sie war wie die anderen Kinder und Brustkind.

Als sie drei Monate alt war, bekam sie eine schwere Erkrankung (der Schaum stand ihr vor dem Mund, der Arzt ordnete ein warmes Bad von Sauerteig an); darauf bekam sie nasse Tücher auf den Kopf und Senfumschläge an den Beinen. Sofort danach war bemerkbar, daß das Kind anders war. Es konnte nicht mehr saugen, die Brustwarze nicht mehr finden, war in jeder Hinsicht idiotisch, bekam spät Zähne, welche sofort wieder ausfielen, konnte nicht lernen.

Laufen lemte sie erst als sie 20 Jahre alt war.

Das Kind wuchs absolut nicht; als es 12 Jahre alt war, saß es noch im Kinderstuhl und war nicht größer als ein Kind von einigen Jahren.

Für die Familie auffallend war es, daB die Ellbogen und Knie so gedreht waren und $d a B$ der Kopf so lange weich geblieben, so da $B$ man wohl "den Finger hineinstecken konnte".

Wie von der Mutter mitgeteilt wurde, wurde von dem Arzte schwere Rachitis angenommen.

Als sie 13 Jahre alt war, wurde sie auf etwa 5 Monate im jüdischen Krankenhaus in Amsterdam aufgenommen. Aus den Notizen, welche man so freundlich war, uns zuzuschicken, folgt, daß das Kind für sein Alter sehr klein war, einen ziemlich kleinen Kopf und einen kurzen, breiten Nacken zeigte. Auch hatte es eine große Zunge, welche meistens außerhalb des Mundes hervortrat. Es war sehr lebhaft, meist munter, versuchte die Aufmerksamkeit dadurch auf sich zu lenken, daß es an den Kleidern des Arztes zog, versuchte Worte zu sagen, brachte es aber nicht weiter als bis zum Hervorbringen unartikulierter Laute. Es war sehr unreinlich.

Hierauf wurde die Patientin bis zum 27. Jahr zu Hause gepflegt. Sie blieb immer zurück. Erst als sie 20 Jahre alt war oder wahrscheinlich noch später, soll sie zu wachsen angefangen haben; nach dieser Zeit wurde sie auch etwas vernünftiger.

Die Menstruation und sexuelle Beharung zeigten sich sehr spät. Genau konnte es nicht angegeben werden, aber unbedingt nicht vor 
ihrem 26. Jahre. Die Menstruation war sehr unregelmäßig, etwa alle 6 oder 7 Monate, und war sehr spärlich (jetzt ist sie regelmäBig, sehr schwach). Schließlich wurde noch mitgeteilt, daß sie immer viele Wunden hatte, zumeist um den Anus.

Status praesens: Sehr kleine Patientin, stehend 1,24 m hoch, mit lebhafter Miene; fortwährend klatscht sie in die Hände oder trampelt mit den Füßen, lacht und bringt unartikulierte Laute hervor. Hierbei ist die Intonation sehr verschieden und abhängig von ihrer Laune, welohe meist munter und froh ist.

Durch Winken, Zeigen und andere Gebärden weiß sie sich zu verständigen. Die Kombinationsfähigkeit besteht, obwohl mangelhaft.

Öfters simuliert sie Krankheit, wenn sie wünscht, zu Bett zu bleiben. Sie winkt dann entweder die Krankenschwester oder den Arzt, zu ihr zu kommen, nimmt dessen Hand und bringt diese an ihre Stirn, hierbei eine sehr betrübte Miene machend. Ist ihr dies gelungen, so entblößt sie ihre Brust, nimmt Hammer und Stethoskop aus der Tasche des Arztes und macht ihm deutlich, daß er sie untersuchen soll. Es fehlt aber die feinere Kombinationsfähigkeit; sie pflegt z. B. das Stethoskop falsch anzufassen oder an ihren Nacken anzulegen.

Gedächtnis besteht; in welchem Umfange es aber vorhanden ist, läßt sich schwierig feststellen.

Personen aus ihrer Umgebung kennt sie, auch kann man öfters sehen, daß sie Personen von früher wiedererkennt.

Dies ist aber schwierig mit Sicherheit zu konstatieren wegen ihrer mangelhaften Fähigkeit sich auszudrücken und wegen ihrer Spontaneität bei jedem Besuch.

Ihre Aufmerksamkeit zu erregen ist schwierig, zumal wenn sie mit etwas beschäftigt ist, daher kommt es denn auch, daß sie die ihr gegebenen Befehle das eine Mal gut, das andere Mal sehr schlecht befolgt, nach Gebärden besser als nach mündlichem Befehl. Bestimmt aber ist es, daß sie einem komplizierten Gespräch gar nicht folgt oder folgen kann, daß sie einigermaßen komplizierte Befehle nicht versteht und daß sehr einfache Fragen oder Aufgaben öfters nur teilweise und jedenfalls mangelhaft verstanden werden. Wenn sie mit etwas einverstanden ist, zeigt sie dies durch sanfte Laute, wenn nicht, so fängt sie an laut zu schreien. Freude zeigt sie meist durch Trampeln mit den Füßen oder durch Händeklatschen. Auch ruft sie dann mittels bestimmter Laute, welche von der Krankenschwester und den Patienten ihrer Abteilung meistens auch verstanden werden, bestimmte Personen zu sich, um diese ihre Freude teilen zu lassen. Eine richtige Arbeit bringt sie nicht fertig. Weil sie aber eine große Neigung hat, alles nachzuahmen. so wendet sie öfters ungeschickte Versuche zu verschiedener Arbeit an, was einen komischen Eindruck macht; und doch wundert man sich, wie weit sie es hierbei noch bringt.

Ohne daß es sie gelehrt ist, kann sie zusammengesetzte Handlungen in die einzelnen Teilhandlungen zerlegen. So weiß sie z. B. ganz genau, daß zum Nähen Nadel und Zwirn notwendig sind, daß auch ein Fingerhut dazu gehört und durch Nähbewegungen weiß sie auch kennbar zu machen, daß sie das Äußerliche der Handlung kennt.

Gibt man ihr die nötigen Utensilien, so nimmt sie diese zur Hand wie eine, die hiermit Bescheid weiß. Tatsächlich kommt aber im großen ganzen nichts zustande. Viele Versuche, ihr etwas beizubringen, sind bis jetzt nicht gelungen.

Die körperliche Untersuchung lehrt folgendes:

Körpermaße: Die Dickenmaße sind gemessen mit dem Bandmaß, die Längenmaße mit dem Zirkel. Um so genau wie möglich zu sein, habe ich die Messungen öfters vorgenommen und habe aus den verschiedenen Werten den 
mittleren berechnet. Es besteht eine fast vollkommene Symmetrie. Unterschiede sind höchstens $1 / 2-3 / 4 \mathrm{~cm}$; ich habe mich also begnügt, die Maße für jeden Körperteil nur einmal anzugeben.

Länge (stehend, Genua valga) . . . . . . . . . . . 124,0 cm

Länge (liegend) . . . . . . . . . . . . . . . . . 127,5,

Spannweite . . . . . . . . . . . . . . . 126,0,

Abstand zwischen Spina ant. sup. und Malleolus internus . . . . . 58,8,

Abstand zwischen Spina ant. sup. und Malleolus externus . . . . 59,5 ",

Abstand zwischen Spina ant. sup. und unterer Patellarkante. . . . 33,2 ,"

Abstand zwischen medialer Kniegelenkspalte und Malleolus internus . 26,1 ,"

Abstand zwischen lateraler Kniegelenkspalte und Malleolus externus . 26,9 ,,

Fußlänge (größte Länge) . . . . . . . . . . . . 17,0,

Fußbreite . . . . . . . . . . . . . . . . 8, 8,0

Fußumfang . . . . . . . . . . . . . . . . . . 19,5,

Umfang des Unterschenkels über den Malleoli . . . . . . . . 18,0 "

Umfang des Unterschenkels $10 \mathrm{~cm}$ unter der Patella . . . . . . 28,2 ",

Umfang des Oberschenkels $10 \mathrm{~cm}$ über der Patella . . . . . . . 44,5 ,

Umfang des Oberschenkels in der Leistenfalte . . . . . . . 55,0 ",

Distantia spinarum. . . . . . . . . . . . . . . . . . . . . 19,2,

Distantia cristarum . . . . . . . . . . . . . 20,5,

Umfang der Hüften . . . . . . . . . . . . . . . . . . . 85,5,

Umfang des Bauches (über dem Nabel) . . . . . . . . . . . 68,5 ,

Umfang der Brust (unter den Mammae) . . . . . . . . . . 79,5 ",

Umfang der Brust (unter den Achseln) . . . . . . . . . . . 78,5 ,

Distantia bitroch. humeri. . . . . . . . . . . . . . . 32,0,

Distantia man. sterni-Proc. spinos. C. VII . . . . . . . . . . . . 11,0,

Distantia Proc. xyphoid.-Proc. spinos. D. VII. . . . . . . . . . . 19,0,

Abstand zwischen Manubrium sterni und Symphysis . . . . . . 43.0 ",

Abstand zwischen Manubrium sterni und Proc. xyphoides . . . . 16,6 ",

Abstand zwischen Proc. xyphoides und Nabel . . . . . . . . . 14,9,

Abstand zwischen Nabel und Symphysis . . . . . . . . . . 12,0,

Länge der Clavicula . . . . . . . . . . . . . . $\pm 10,0$,

Abstand zwischen Akromion und Radiusköpfehen . . . . . . . . 22,1,

Abstand zwischen Radiusköpfchen und Handgelenk . . . . . . . 17,5 "

Länge der Hand (Pulsgelenk bis Ende Mittelfinger) . . . . . . . . 13,1 ,

Mittelfinger . . . . . . . . . . . . . . . . 7,3,

Zeigefinger . . . . . . . . . . . . . 6,5,

Ringfinger. . . . . . . . . . . . . . . . . 6,6"

Kleiner Finger. . . . . . . . . . . . . . . . . . . . . . 5,1,

Daumen. . . . . . . . . . . . . . . . . . 4 4,4,

Breite der Hand . . . . . . . . . . . . . . . . . . . . 16,5 ",

Umfang des Oberarmes (10 cm unter dem Akromion) . . . . . . . 24,5,

Umfang des Unterarmes (dickster Teil) . . . . . . . . . . . . 20,5,

Umfang der Faust (Daumen innenwärts) . . . . . . . . . 21,5 "

Kopfumfang ................... 54,5,

Distantia ant. post. maxima . . . . . . . . . . . . . . . . 10,2,

Distantia transversa maxima . . . . . . . . . . . . . 14,8 ,

Distantia bizygomatica . . . . . . . . . . . . . . . 11,0,

Höhe des Antlitzes (Kinn bis Haarwuchs) . . . . . . . . . . . . 15,5 ,

Höhe des Antlitzes (Kinn bis Glabella) . . . . . . . . . . . . . 10,0,

Distantia mento-auricularis . . . . . . . . . . . . . . . . 13,0,

Distantia bimandibularis . . . . . . . . . . . . 10,5, 
Bei der Inspektion zeigt sich sofort Verschiedenes. Die ausgesprochenen Genua valga sind die Ursache, daß Patientin liegend viel größer ist als stehend.

Die Patientin hat eine wachsartige, anämische Hautfarbe.

Es besteht eine starke Obesitas, am deutlichsten um die Hüften und die Nates, aber auch an den Knöcheln und den Unterarmen, an Händen und Füßen (s. Fig. 1 bis 3).

Ihr Antlitz aber kann man eher mager als dick nennen.

Diese Obesitas an den Oberschenkeln und den Hüften gibt dem Individuum, zumal kombiniert mit dem eigentümlichen Stand seiner Extremitäten, ein unförmliches Aussehen.

Auffallend ist die kräftige Behaarung der äußeren Genitalien, welche sich auf der Innenseite der Oberschenkel fortsetzt, der üppige Haarwuchs am Kopf, dessen Implantationslinie weit auf die Stirn übergreift, der kräftige Haarwuchs in den Achselhöhlen. Die Haare selber sind sehr dick und hart und dicht gepflanzt.

Die sekundären Geschlechtscharaktere sind sehr kräftig entwickelt. Die Mammae sind groß, mit deutlich fühlbarem Drüsengewebe.

Die Haut ist trocken und zeigt, zumal auf der Hand- und Fußrückenseite, eine eigentümliche Abweichung.

Die Haut ist hier so rauh, daß Hände und FüBe, wiederholter Reinigung ungeachtet, schmutzig aussehen. Die Haut der Hände gleicht der einer Wäscherin (Einreibung mit Glycerin hat nichts genützt).

Der Kopf ist kurz und brachycephal. Der Scheitel des Antlitzes ist klein und bildet einen großen Kontrast zu dem außerordentlich großen Unterkiefer.

Der Schädel zeigt eine Einsenkung zwischen den beiden Tubera parietalia, welche hierdurch sehr deutlich als einzelne Beulen zu fühlen sind (Caput natiforme). Der Hinterkopf ist flach. Die Stirn zeigt eher eine quadratische als eine runde Form.

Die Ohren sind äußerst klein. Die Patientin hört gut.

Die Nase ist auffallend groß und vorspringend.

Die Augen haben eine graue Farbe. Die Pupillen reagieren auf Licht und Konvergenz. Die rechte Pupille ist aber nicht rund; sie ist verzogen nach dem unteren inneren Augenwinkel. Patientin hat eine Macula corneae.

Der Visus ist normal. Die Papilla nervi optici zeigt keine deutlichen Abweichungen (spezialistische Untersuchung). Die Augenbewegungen sind intakt.

Sie hat ein sehr unvollkommenes Gebiß mit cariösen Zähnen und Backzähnen. Reste eines Milchgebisses sind nicht bemerkbar. Der Gaumen ist gewölbt, aber nicht sehr stark. Der Oberkiefer ist sehr kurz und schmal, der Unterkiefer, wie gesagt, außerordentlich groß, breit und vorspringend.

Die Zunge ist sehr groß und hängt öfters teilweise aus dem Munde. Die Bewegungen sind gut. Auch die Kieferbewegungen sind intakt. In der Facialismuskulatur sind keine Abweichungen, keine adenoiden Vegetationen.

Durch die große Nase, den kolossalen Unterkiefer mit großer Zunge hat unsere Patientin ein akromegales Gesicht.

Im Gegensatz hierzu sind die Arme zu kurz im Verhältnis zum Rumpf, was direkt nachzuweisen ist, wenn wir die angegebenen Maße mit denjenigen eines normalen Typus vergleichen.

Das Längenverhältnis zwischen Oberarm, Unterarm und Hand ist aber vollkommen normal (s. später die ausführlichen Betrachtungen).

Für die Beine gilt genau dasselbe wie für die Arme.

Patientin hat Cubiti valgi und stark ausgesprochene Genua valga und Pedes valgi. 
nebst Bemerkungen über die Klassifikation der Zwerge.

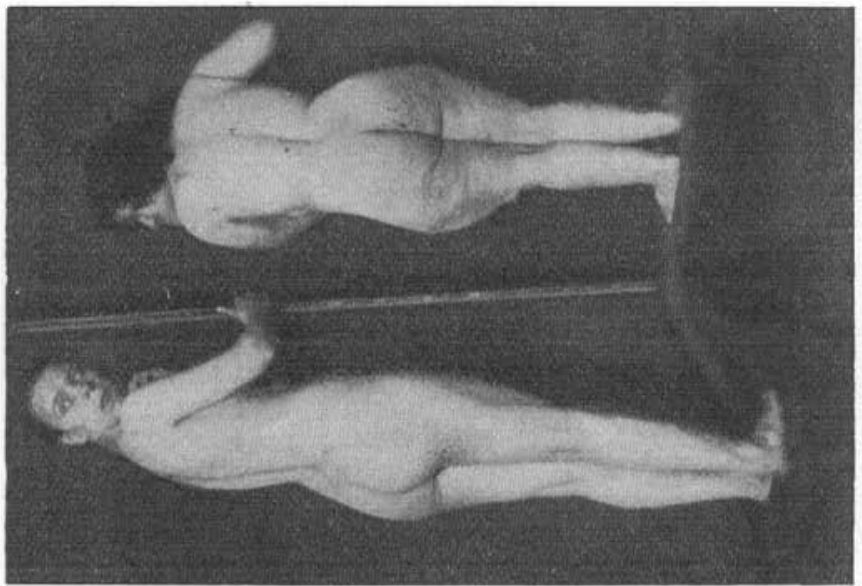

츤

s

栗

్ㅐㅇ

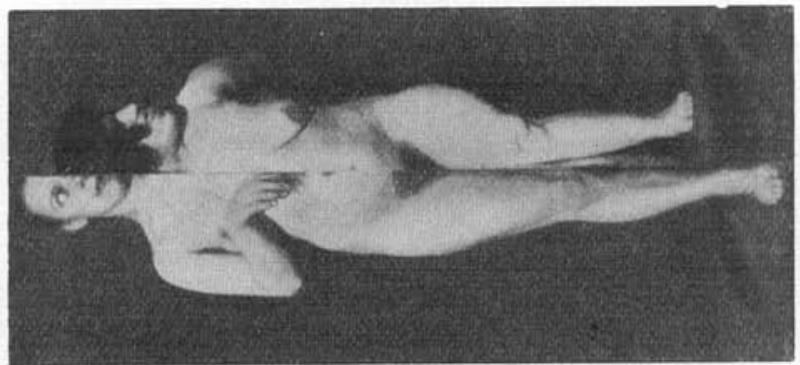

i

플

ह

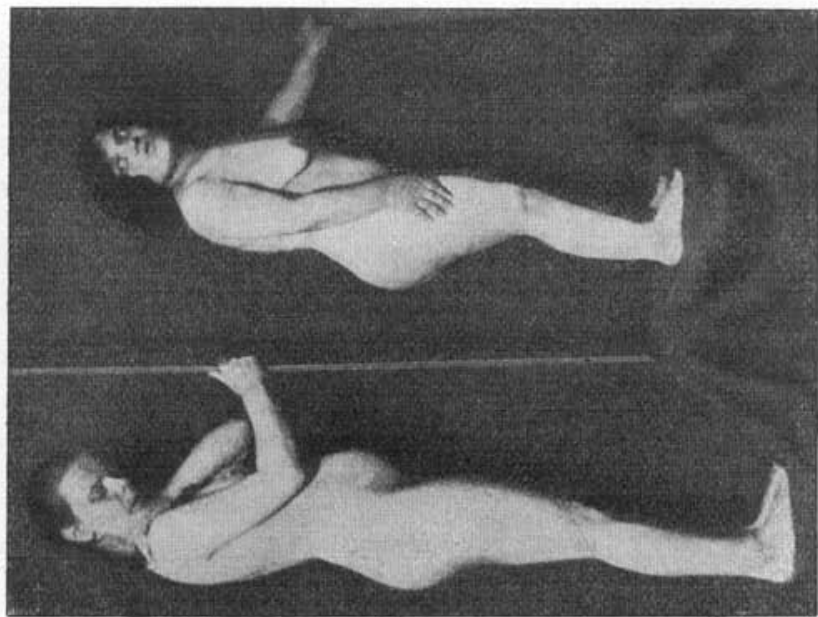

है

i

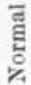


Der Unterschenkel ist nach außen rotiert. Die Patella sieht nach außen,

Die Hautveränderung und Obesitas habe ich schon besprochen.

Auffallende Verkrümmungen sind nicht vorhanden. Die Unterarme sind aber etwas mehr gebogen als gewöhnlich.

Im ganzen ist die Muskulatur schlaff und sehr schlecht entwickelt. Daher kommt es denn auch, daß die Kraft der Arm- und Beinmuskeln äußerst gering ist; die koordinatorischen Bewegungen geschehen aber gut. Ihr Gehen ist sehr mangelhaft, breitspurig. Sie läuft mit kleinen Schritten und hält hierbei die Arme in starker Abduction. Hierbei werden die Füße nur wenig gehoben. Sie läuft auf den Fersen, nicht auf den Zehen.

Auffallend ist eine Schlaffheit der Gelenkbänder. In allen Gelenken kann man eine Anzahl von ausgiebigen Bewegungen machen, so daß man Patientin in allerlei, dem Normalen unmöglichen Stellungen, mit großer Bequemlichkeit bringen kann.

Während nun einerseits eine ausgesprochene Schlaffheit herrscht, fällt die Rigidität an den Beinen auf bei passiven Bewegungen; bei jeder Bewegung werden die Muskeln sehr steif gespannt.

Bei jeder passiven Bewegung, zumeist bei plötzlicher, fällt ein stärkerer Widerstand auf wie bei einer Knippfeder.

Die Sehnenreflexe sind an Armen und Beinen lebhaft, zumeist aber an den Beinen. Dann und wann kann man einen deutlichen Fußklonus wahrnehmen.

Die Bauchreflexe sind rechts $=$ links lebhaft.

Der Fußsohlenreflex ist meines Erachtens als im Sinne des Babinskireflexes aufzufassen. Die Untersuchung macht bei ihr öfters große Schwierigkeiten, weil sie sofort, wenn man ihre Fußsohle berührt oder nur auf sie hinzeigt, mancherlei Mitbewegungen ausführt, nicht nur mit den Füßen, sondern auch mit den Händen. Nach mehreren Untersuchungen, wobei versucht wurde, ihre Aufmerksamkeit abzulenken, kam ich zu der Uberzeugung, daß Babinski-Fußsohlenreflex vorhanden war. in dieser Auffassung wurde ich bestärkt, weil in der Krankengeschichte aus dem „Wilhelmina Gasthuis" Babinski als positiv angegeben steht.

Herz und Lungen zeigen keine Abweichungen. Auch die Bauchorgane nicht.

Genitalia interna (Untersuchung von Dr. Götte, Gynäkolog, Haarlem): Portio sehr klein. Der Uterus liegt gestreckt, zeigt keinen Knick und hat die Größe eines Mädchenuterus (fötale Form und infantile Größe). Die Ovarien sind sehr klein und fast nicht zu fühlen. Der Urin enthält keinen Zucker und kein Albumen.

Es ist keine Polyurie vorhanden, obwohl im Verhältnis zu der Quantität, die sie trinkt, das Quantum ziemlich groß ist. Meistens ein sehr niedriges spezifisches Gewicht.

Die Temperatur ist etwas unter der Norm. Der Puls ist meist klein und sehr schwach. Die Spannung ist gering.

Nach $200 \mathrm{~g}$ Dextrose per os kein Zucker in dem Urin.

Auf $200 \mathrm{~g}$ Dextrose und $1 \mathrm{ccm}$ einer Adrenalinlösung $1: 1000$ nach einer Stunde eine sehr deutliche positive Zuckerreaktion (12. Juni 1913).

Sie menstruiert regelmäßig, aber sehr schwach.

Auffallend ist, daß der Gesundheitszustand der Patientin sehr wechselt, daB sie öfters über Kopfschmerzen klagt und auch öfters erbricht, zumeist gegen Abend.

Seit dem Niederschreiben dieses Status ist unsere Patientin meistens im Bett verpflegt worden.

Während sie bei ihrer Aufnahme 108 Pfund wog, wiegt sie jetzt nur noch 70 Pfund.

Ihre ausgesprochene Obesitas ist größtenteils verschwunden. 
Röntgenologische Untersuchung.

Es wurden Röntgenogramme angefertigt von der Schädelbasis, von der Hand, von Ellbogen, Fuß, Unterschenkel, Knie und Hüfte (Tafel I-IV). Die große Versatilität der Patientin nötigte uns zu Momentaufnahmen ${ }^{1}$ ).

Schädelbasis: Keine Abweichungen der Knochen und der Nähte. Abflachung der Sella turcica ohne Vergrößerung in sagittaler Richtung.

Hand und Handgelenk (dorsovolare Aufnahme): Graziler Knochenbau, besonders am Metacarpus, namentlich Metacarpale IV. Der Carpus erscheint sehr schmal dadurch, daß die Carpalia sich mehr als normal bedecken. Die Epiphysärlinien sind verstrichen. Nur am Radius ist vielleicht noch ein schmaler Rest angedeutet. Keine Spur von Verbreiterung der Epiphysen.

Die Ulna zeigt eine geringe Verkrümmung, $2-3 \mathrm{~cm}$ vom distalen Ende entfernt und ulnarwärts konvex.

Ellbogen: Geringe Verkrümmung des Humerus. Epiphysen nicht vergrößert, $3 \mathrm{~cm}$ vom Radiusköpfchen entfernt eine deutliche Verbreiterung der Diaphyse, spindelförmig, besonders auf der ulnaren Seite. An der Stelle ist auch die Diaphyse dunkler, ein Hinweis auf das Zirkuläre des Prozesses.

Fuß und unterer Teil des Unterschenkels (Seitenaufnahme mit der Röhre etwas nach hinten zur Erhaltung eines freien Bildes des Malleolus externus): Auffallende Grazilität der Knochen, auffallend kleiner Talus, Calcaneus und Tarsalia. Keine geschwollenen Epiphysen. Epiphysärlinien verstrichen. Die Fibula zeigt eine bedeutende Verdickung, zweifingerbreit oberhalb des Malleolus anfangend, so daß vier Finger höher die Fibula zwei Drittel der Dicke hat wie die Tibia. Es besteht eine geringe Verkrümmung der Fibula.

Kniegelenk: Seitenaufnahme, wie die Konfiguration der Weichteile deutlich macht. Das Skelett des Oberschenkels und der Kniescheibe würde eine Aufnahme von vorn nach hinten vortäuschen. Die Kniescheibe, die vor dem Femur zu liegen hat, liegt nur zu einem Drittel frei.

Der Unterschenkel ist tordiert.

Die Gelenkspalte ist weit. Das Fibulaköpfchen ist verbreitert. Die Epiphyse des Femurs ist im Verhältnis zur Diaphyse ziemlich groß. Die Fibula zeigt zweifingerbreit unter dem Capitulum eine Verbreiterung ihres Schattens, die auf periostale Verdickung hinweist, dort besteht auch eine gringe Verkrümmung mit der Konvexität nach außen.

Hüftgelenk: Sehr weite Gelenkspalte. Auffällig graziler Knochenbau. Der Femurkopf ist sehr klein.

Zusammenfassung: Epiphysärlinien verstrichen. Auffällig graziler Knochenbau. Keine verdickten Epiphysen, mit Ausnahme des Capitulum fibulae und vielleicht der Femurkondylen. Minimale, aber unverkennbare Verkrümmung der langen Röhrenknochen. Periostale, spindelförmige Verdickung der Fibula und des Radius.

\section{Zusammenfassung:}

Unsere Patientin ist ein Zwerg mit den folgenden auffallenden Erscheinungen :

1. Akromegaler Gesichtsausdruck - große Zunge und kolossale Unterkiefer.

1) Dr. L. Heil bro n war so liebenswürdig, im Röntgenologischen Laboratorium des Herrn Professor Wertheim Salomonson in Amsterdam die verschiedenen Aufnahmen für mich herzustcllen. Beiden sage ich an dieser Stelle meinen aufrichtigen Dank. 
2. Mikromele Verkürzung der Extremitäten mit normalem Längenverhältnis von Hand, Unter- und Oberarm resp. Fuß, Unter- und Oberschenkel (s. später).

3. Alabasterfarbene Haut, die auf Hand- und Fußrücken eigentümliche Störungen zeigt.

4. Sehr wechselnde Obesitas mit Prädilektion für die Nates und die Mammae.

5. Geringe Entwicklung der Genitalia interna, verspätetes Auftreten der Menstruation, die jetzt regelmäßig ist; dagegen stark ausgebildete sekundäre Geschlechtscharaktere.

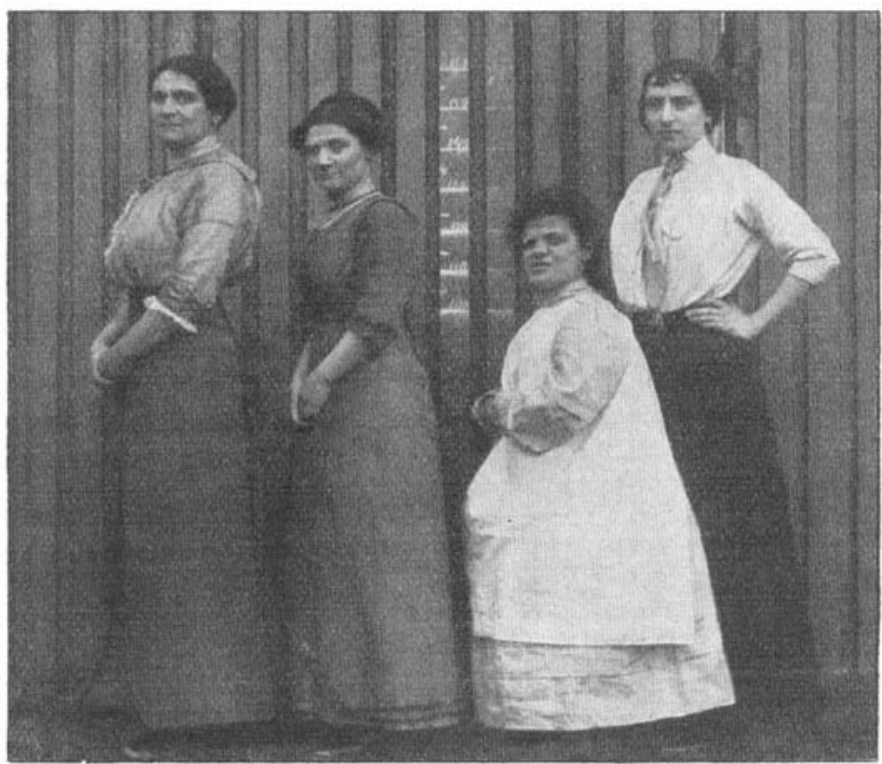

Fig. 4. Die Kranke und ihre drei normalen Schwestern.

6. Sehr schlaffe und wenig kräftige Muskeln, mit äußerst schlaffen Gelenkbändern; erhöhte Sehnenreflexe und ungeachtet der Schlaffheit spastische Erscheinungen, Babinski, Klonus (?).

7. Kopfschmerz, häufiges Erbrechen.

8. Knochensystem, röntgenologisch untersucht:

a) ohne ausgesprochene Verkrümmungen.

b) geschlossene Epiphysenscheiben.

c) Auftreibung des Periosts am Radius und unregelmäßige (periostale) Verdickung der Fibula mit unerheblicher Verkrümmung.

d) Grazilität aller Knochen.

e) Sella turcica flach, wahrscheinlich normaler Größe. 
9. Positive Wassermann-Reaktion, Macula corneae. Narben am Anus und auf den Nates.

10. Gehört zu den versatilen Idioten.

Wenn wir versuchen, unseren Fall in einer bestimmten Gruppe von Zwergwuchs unterzubringen, so begegnen wir vielen Schwierigkeiten.

Wie wir schon kurz dargetan haben, spielen die Körperproportionen bei der Abgrenzung der verschiedenen Formen eine große Rolle. Auch wird hierbei dem Verhältnis zwischen den Weichteilen und dem Skelett Rechnung getragen.

is:

In unserem Falle haben wir nicht nur ein Mißverhältnis der Körperproportionen, sondern auch zwischen den Weichteilen und den Knochen.

In unserem Falle ist der Rumpf zwar zu klein, jedoch sind im Verhältnis zu ihm die Extremitäten viel zu kurz.

Es ist eine deutlich ausgesprochene Mikromelie der Extremitäten vorhanden.

Weil Heredität und Rasse von großem Einfluß auf die verschiedenen Körperproportionen sind, habe ich die verschiedenen Maße unserer $\mathrm{Pa}$ tientin verglichen mit denen der versehiedenen Körperteile ihrer drei Schwestern, von 27, 32 und 39 Jahren resp. (Siehe Fig. 4.)

Dies, um so genau wie möglich die Art der Verkleinerung festzustellen ${ }^{1}$ ).

1) Wie vorsichtig man sein muß mit bestimmten Folgerungen, möge sich aus folgendem erweisen.

Als ich die Maße der verschiedenen Körperteile unserer Patientin verglich mit dem mittleren Werte derselben Maße, welche ich durch Messung von 16 weiblichen Patienten meiner Abteilung bekommen hatte, zeigte sich eine sehr eigentümliche Verkürzung der Extremitäten. Außer daß der Rumpf zu klein war, waren auch die Extremitäten im Verhältnis zum Rumpf viel zu kurz. Es bestand Mikromelie. Es war aber nicht nur eine Mikromelie vorhanden, sondern die Verkürzung war am größten in den distalen Teilen, zumal an den Hände. Ich fand folgende Verkürzungsverhältnisse:

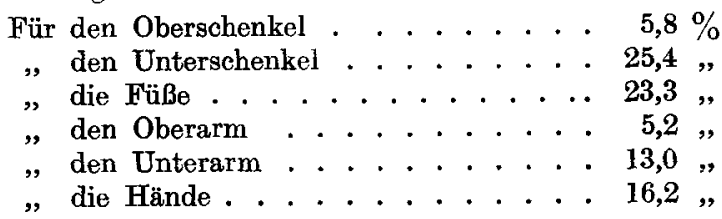

Auf diesem Resultat fußend, sprach ich am 4. November 1913 in der Neurologenversammlung in Amsterdam, wo ich die Patientin vorstellte, von Micromelia. a cromelica im Gegensatz zu der Micromelia rhizomelica. Dies im Zusammenhang mit der zentralen Störung und den anderen Vorgängen, die auf Hypophysisleiden hinweisen (s. später), der Kontrast mit der Megalomelia akromelica oder Akromegalia, stärkten mich in der Auffassung, daß eine Hypophysisstörung in diesem Falle eine große Rolle spielte.

Ich hatte aber die Rassen- und ev. hereditäre Eigenart zu sehr vernachlässigt. Die 16 weiblichen Patienten sind nümlich Christinnen, unsere Patientin ist eine Jüdin. Nach Vergleich mit den Maßen ihrer drei Schwestern blieb von der vorausgesetzten Mikromelia acromelica nichts übrig (s. später). 
Ich lasse hier die Tabelle der verschiedenen Körpermaße folgen.

\begin{tabular}{|c|c|c|c|c|}
\hline & & & $27 \mathrm{~J}$. & $\mathrm{n} \mathrm{cm}$ \\
\hline . & 152,1 & 144, & 152,5 & 27,5 \\
\hline bst & 78,0 & 70,3 & 77,5 & \\
\hline nt. sup. & & & & \\
\hline vischen Spin. ant. sup. und Mal & 80,2 & 73,5 & 80,5 & 59,5 \\
\hline 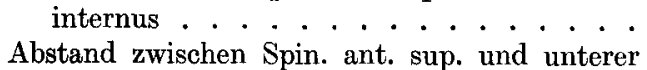 & 78,5 & 73,0 & 79,2 & 58,8 \\
\hline larkante ... . . . . . . & 42,8 & 41,9 & 43,0 & 33,2 \\
\hline $\begin{array}{l}\text { bstand zwischen Symphysis und Malleolus in- } \\
\text { ternus } . . .\end{array}$ & 72,5 & 67,7 & 72,0 & 52,5 \\
\hline 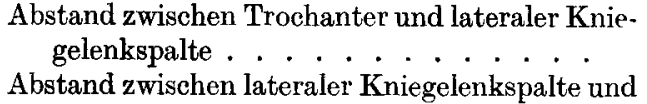 & 41,3 & 37,0 & 40,8 & 34,5 \\
\hline & 36,2 & 32,5 & 35,6 & 26,9 \\
\hline $\begin{array}{l}\text { aedialer Kniegelenk } \\
\text { us. . . . . . . . . }\end{array}$ & 35,8 & 31,4 & 34,5 & 26,1 \\
\hline 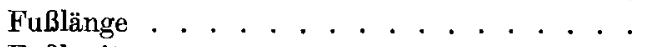 & 22,3 & 20,6 & 22,4 & 17,0 \\
\hline e. . & 8 & 8,1 & 8,6 & 8,0 \\
\hline ußumfang . . & 21,0 & 19,5 & 21,0 & 19,5 \\
\hline mfang des Unterschenkels $10 \mathrm{~cm}$ unter der & & & & \\
\hline ........ & 34,5 & 32,0 & 30,5 & 28,2 \\
\hline s über den Malleoli & 21,5 & 19,5 & 19,5 & 18,0 \\
\hline $\begin{array}{l}\mathrm{cm} \text { über der Pa- } \\
. . . . . . .\end{array}$ & 44,5 & & & 44,0 \\
\hline els in der Leistenfalte & 54,0 & & 0 & 55,0 \\
\hline 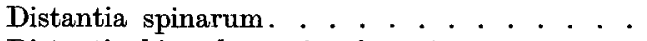 & 24,5 & & 2 & 19,2 \\
\hline terica femoris . . & 31 & & 2 & $?$ \\
\hline 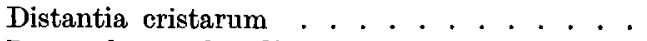 & 28,2 & 25,2 & 24,0 & 20,5 \\
\hline n Zehe : . . . . & 6,7 & 6,2 & 6,5 & 4,5 \\
\hline $\begin{array}{l}\text { bstand zwischen Akromion und Radiusköpt- } \\
\text { chen }\end{array}$ & 28,2 & 25,1 & 28,5 & 22,1 \\
\hline 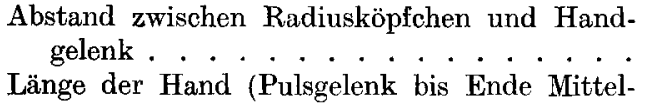 & 22,0 & 19,5 & 21,7 & 17,5 \\
\hline & 16,8 & 14,7 & 16,8 & 13,1 \\
\hline Länge & 9 & & 9,1 & 7,3 \\
\hline Finger. . & $7, \mathrm{C}$ & 8 & 6,7 & 5,1 \\
\hline Läng & 6,6 & 5,5 & 6,4 & 4,4 \\
\hline Läng & 8,5 & 7,3 & 7,8 & 6,5 \\
\hline Länge & 9,0 & 7,4 & 8,5 & 6,6 \\
\hline Arme & 64,7 & 58,3 & 63,2 & 49,5 \\
\hline 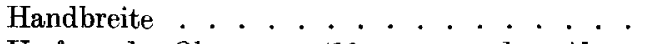 & 20,0 & $17,5-18$ & 17,5 & 16,5 \\
\hline 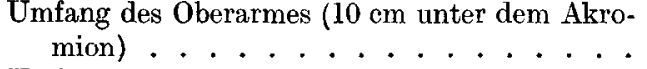 & 20 & & 21,0 & 24,5 \\
\hline des Unterarms & 23,5 & 22,5 & 21,0 & 20,5 \\
\hline $\begin{array}{l}\text { Umfang der Faust (Daumen einwärts) } \\
\text { Abstand zwischen Manubrium sterni und Syn }\end{array}$ & 25,0 & 20,5 & 22,2 & 21,8 \\
\hline physis.. & 46,2 & 41,9 & 47,8 & 43,0 \\
\hline
\end{tabular}




\begin{tabular}{|c|c|c|c|c|}
\hline & $\begin{array}{c}39 \mathrm{~J} . \\
\mathrm{cm}\end{array}$ & $\begin{array}{c}32 \mathrm{~J} . \\
\mathrm{cm}\end{array}$ & $27 \mathrm{~J}$. & $\begin{array}{l}\text { Pation- } \\
\text { tin om }\end{array}$ \\
\hline $\begin{array}{l}\text { Abstand zwischen Manubrium sterni und Proc. } \\
\text { xyphoides . . . . . . . . . . . . }\end{array}$ & 18,2 & 15,8 & 18,2 & 16,6 \\
\hline Abstand zwischen Proc. xyphoides und Nabel. & 15,0 & 14,3 & 13,0 & 14,9 \\
\hline Abstand zwischen Nabel und Symphysis . : & 14.1 & 12,1 & 16,2 & 12,0 \\
\hline Kopfumfang . . . . . . . . . . & 54,2 & 52,5 & 54,5 & 54,5 \\
\hline Distantia biparietalis . & 14,5 & 14,4 & 14,3 & 14,8 \\
\hline
\end{tabular}

Aus dieser Tabelle geht das Folgende hervor. Die Verkleinerung der Rumpflänge ist nur wenig ausgesprochen. $\mathrm{Ja}$, bei der kleinsten der drei gut proportionierten Schwestern fand ich eine kleinere Rumpflänge als bei unserer Patientin.

Die Verkürzung der Extremitäten im Verhältnis zum Rumpf zeigt sich aber sehr deutlich. Man findet nämlich:

Obere Extremitäten . . . . . . . . . . $\pm 15 \%$ zu kurz. Untere Extremitäten. . . . . . . . . . $\pm 18 \%$ zu kurz.

Ausgerechnet für die verschiedenen Unterteile findet man:

für den Oberschenkel! . . . . . . . . . . . . 15,3\%

für den Unterschenkel . . . . . . . . . . . . 18,4\%

für die Füße . . . . . . . . . . . . . . . 17,8\%

für den Oberarm . . . . . . . . . . . . . . $16,6 \%$

für den Unterarm . . . . . . . . . . . . . 13,4\%

für die Hände. . . . . . . . . . . . . . . . . . . . . 14,2\%

Für die oberen und unteren Extremitäten gegenseitig also nur derart kleine Unterschiede, daß diese sehr gut erklärt werden können durch kleine Meßfehler, die wohl nicht zu umgehen sind.

Die mikromele Verkürzung ist also für die verschiedenen Extremitätensegmente dieselbe $\mathbf{1}$ ).

Wie man auch bei kleinen Kindern sozusagen eine physiologische Mikromelia findet im Vergleich mit dem erwachsenen Individuum, so wäre es wohl möglich, daß wir es hier mit kindlichen Proportionen zu tun hätten.

Dies ist nun nicht der Fall.

Nach den Untersuchungen von Lange wachsen nach der Geburt die Extremitäten (und zwar die zentralsten Segmente) am kräftigsten.

1) Bei diesen Berechnungen habe ich den Abstand von der Incisura sterni bis zur Symphysis als Vergleichsmaß angenommen. Öfters werden die verschiedenen Maße, die man findet, verglichen mit der ganzen Länge des Individuums. Hier. durch macht man den Fehler (z. B. bei zu kurzen Extremitäten und normalem Rumpf), einen Vergleich zu ziehen mit einem normalen und einem abnormalen Maß, also mit der Summe zweier ungleichmäßiger Größen, und kommt also zu falschen Resultaten.

Z. f. d. g. Neur. u. Psych. O. XXXII. 
Weil während des intrauterinen Lebens sich die relative Länge der Hand- und Fußwurzel vergrößert, wobei die relative Länge der oberen Segmente abnimmt, liegen nach der Geburt die Verhältnisse umgekehrt. Hand- und Fußwurzel lassen immer mehr im Wachstum nach, während Oberschenkel und Oberarm länger werden (zitiert nach Gundobin, Die Besonderheiten des Kindesalters. Berlin 1912).

Es ergibt sich nun die interessante Tatsache, daß das Verhältnis zwischen Oberarm, Unterarm und Hand, zwischen Oberschenkel, Unterschenkel und $F u B$ bei unserer Patientin ziemlich genau dasselbe wie bei ihren Schwestern ist.

Der Index zwischen Ober- und Unterarm ist bei den Schwestern 49,46, bei unserer Zwergin 49,27.

Der Index zwischen Unterarm und Hand ist bei den Schwestern 74,19, bei unserer Patientin 74,22.

Der Index zwischen Oberschenkel und Unterschenkel ist bei den Schwestern 81,7, bei unserem Zwerg 81 .

Der Index zwischen Unterschenkel und Fuß ist bei den Schwestern 62,3, bei unserem Zwerg 63,2.

Diese interessante Übereinstimmung schließt die Möglichkeit, daß wir es bei unserem $Z$ werg mit kindlichen Proportionen zu tun haben. schon aus.

Auch die Messungen, die ich hierzu bei einigen Judenkindern im Alter von 3 bis zu 42 Monaten vornahm, ergaben andere Durchschnittsindices ${ }^{1}$.

Oberarm-Unterarm 84,7.

Unterarm-Hand 82,6.

Oberschenkel-Unterschenkel 77,2 .

Unterschenkel-Fuß 75.

Wir haben es hier also zu tun mit einem disproportionierten Zwerg mit ausgesprochener mikromeler Verkürzung der Extremitäten, wobei aber das Verhältnis zwischen den verschiedenen Extremitätssegmenten dasselbe ist wie bei seinen erwachsenen normalen Geschwistern.

Aus dem Vorgehenden ist also schon klar, daß wir es hier weder mit essentiellem Zwergwuchs noch mit Ateleiosiszwergwuchs zu tun haben. Denn die Charalkteristica dieses letzteren, die kindliche Physiognomie, die kindlichen Proportionen, das Fehlen von nachweisbaren schädlichen Einflüssen, die normale Intelligenz und die ausgesprochene Heredität, sind in unserem Falle sicherlich nicht vorhanden.

1) Herrn Dr. Schippers, dem Direktor des Kinderkrankenhauses in Amster. dam, spreche ich auch an dieser Stelle meinen Dank aus für die wohlwollende Freundlichkeit, mit der er mir sein Material zur Verfügung stellte. 
Unser Fall gehört also in diejenige Gruppe, wo der Zwergwuchs aufgefaßt werden muß als Symptom.

Die Diagnose Achondroplasie ist meines Erachtens von der Hand zu weisen.

Zwar haben wir eine mikromele Verkürzung der Extremitäten vor uns, diese ist aber ganz anders wie bei der Achondroplasie. Dort ist ja die Verkürzung der Extremitäten meistens in rhizomelem Sinne, was hier absolut nicht der Fall ist. In unserem Falle fällt die genaue Utbereinstimmung im Verhältnis zwischen den verschiedenen Extremitätssegmenten mit derjenigen der Geschwister auf. Dort findet man gewöhnlich Makrocephalie, die von P. Marie zuerst als Charakteristicum hervorgehoben worden ist; den eingesunkenen Nasenrücken, die Verkürzung der Schädelbasis, eine eigentümliche Löffelform der Squama occipitalis, die dorsolumbale Kyphosis, welche bei der Achondroplasie regelmäßig vorkommt; welche Formstörungen von Murk Jansen auf einleuchtende Weise als direkte Folge von mechanischen Kräften, als Mißbildungen rein mechanischen Ursprungs aufgefaßt werden.

Alle diese Charakteristica fehlen bei unserer Patientin. Auch die Röntgenbilder des Skeletts schließen die Achondroplasie aus.

Wir finden keine ausgesprochenen Volumenveränderungen der Epiphysen, ebensowenig die zumeist dicken, groben Diaphysen.

Wir sehen nicht die oft vorkommenden Exostosen. Im Gegenteil trifft man eine Kalkarmut an, die Diaphysen sind schmächtig gebaut.

Die Tibia ist nicht kürzer als die Fibula, eine Erscheinung, welche bei der Achondroplasie außerordentlich häufig angetroffen wird, so daß das Fibulaköpfchen die Tibia überragt.

Das kräftigste Argument wäre aber die Tatsache, daß unsere Patientin bei der Geburt normal groß war.

Dies schließt Achondroplasie aus, die eine exquisit kongenitale Störung ist. Dennoch habe ich die Tatsache nicht allzusehr betont, weil es sich bei ihr um ein anamnestisches Datum handelt. Auch die Tatsache, daß die Achondroplasten in der Regel normale Intelligenz zeigen, macht die Diagnose der Achondroplasie sehr unwahrscheinlich.

Ferner ist der Achondroplast ein gesundes kräftiges Individuum, oft mit athletischer Muskulatur und gesteigerter Sexualität; unsere Patientin nicht. Wohl sind die sekundären Geschlechtscharaktere bei ihr sehr stark entwickelt, aber die Genitalia interna sind in der Entwicklung zurückgeblieben.

Obschon wir also einen disproportionierten mikromelen Zwerg vor uns haben, kann dennoch von Achondroplasie keine Rede sein. Ebensowenig von der Form des rachitischen $Z$ wergwuchses, wo die Kleinheit auf ausgesprochenen Skelettdeformitäten beruht und wo durch die Veränderung des Thorax oder durch starke Verkrümmung 
der unteren Extremitäten ein mißgebildeter Zwerg entsteht, ein Individuum, dessen Arme meist einen zu langen Eindruck machen, und wo die Asymmetrie oft ausgebildet ist (S. von Zimmern und Sterling).

Mit Ausnahme des Schädels darf man in unserem Falle von Skelettverbildungen in grobem Sinne nicht sprechen ebensowenig von Asymmetrie. Die Maße sind rechts und links fast gleich. Ebensowenig sind die plumpen Knochen und die unregelmäßigen Epiphysärverdickungen auf den Röntgenbildern vorhanden.

Dennoch möchte ich die Möglichkeit nicht ganz ausschließen, daß die Rachitis bei unserem Falle eine Rolle gespielt hat, zumal die Rachitis, ohne daß Verkrümmungen der Knochen vorkommen, einen eigentümlichen disproportionierten Zwerg mit relativ langem Rumpf und abnorm kurzen. Extremitäten hervorrufen kann, besonders bei sehr chronischen und schweren Fällen. Wieland gibt hiervon ein schönes Beispiel.

Bouchut wies nach, daß rachitische Kinder während ihrer Krankheit kaum wachsen, und in schweren Fällen soll, wie Feldmann behauptet, auch nach der Ausheilung der Krankheit die Entwicklung gestört bleiben.

Gulecke teilt drei Fälle von Zwergwuchs mit, die in der Jugend schwer rachitisch waren, und wo er eine prämature Synostose der Epiphysärscheiben fand. Er vermutet, daß durch die Rachitis der Epiphysärknorpel schwer lädiert wurde, wodurch die reparatorischen Vorgänge in abnormaler Weise stattfanden und zu frühzeitiger Verknöcherung führten.

Murk Jansen meint, daß die Rachitis als solche ohne Zwischenkunft von Verkümmerungen Zwergwuchs verursachen kann.

Unsere Patientin kann in der Jugend zwar Rachitis gehabt haben. Hierfür wären zahlreiche Tatsachen anzuführen; den anamnestischen Daten kann man natürlich nicht allzu großen Wert beilegen. Wachstumsstörungen in der Jugend werden wohl meistens als rachitische aufgefaßt ohne genügende Beweise.

Die spontane Mitteilung aber, daß der Kopf des Kindes so weich war, daß man ihn mit dem Finger eindrücken konnte, erregt Verdacht auf Rachitis, da doch die Kraniotabes speziell bei der frühen Rachitis auftritt.

Unsere Patientin hat eine ausgesprochene Brachycephalie, eine starke Abplattung des Hinterkopfes, ein Caput natiforme, einen auffallend kleinen Gesichtsschädel. Dies und die Caries der Zähne sind bei der Rachitis häufig vorkommende Symptome, obschon der natiforme Schädel nach Fournier bis jetzt ausschließlich bei Heredoluetikern nachgewiesen ist. 
Auch die abnorme Schlaffheit der Gelenkbänder, die Mitteilung der Mutter, „daß alles beim Kinde gedreht war", die mit dem Röntgenverfahren nachgewiesenen, wenn auch geringfügigen Verkrümmungen der langen Röhrenknochen, könnte man auf eine abgelaufene Rachitis zurückführen. Auch die Kombination mit Idiotie könnte man sogar für diese Auffassung heranziehen. Weygandt nennt Ziehen, Demoor, Bourneville, Comby u. a., die sich für einen rachitischen Schwachsinn aussprechen, und teilt mit, daß Kellner einen neunjährigen Knaben von $104 \mathrm{~cm}$ Länge statt $140 \mathrm{~cm}$ normal beschrieb mit einem Schädelumfang von $51 \mathrm{~cm}$, währenddem Wirbelsäule, Zähne und Extremitäten rachitisch verändert waren, und bei dem ein tiefer Schwachsinn bestand.

(Der Fall Schrumpfs ist in dieser Hinsicht interessant.)

Dagegen zeigt unsere Patientin auch eine große Zahl von Symptomen, die nicht in den Rahmen der Rachitis hineinpassen. Die große Zunge mit dem ausspringenden großen Unterkiefer, die starke Adipositas, das Zurückbleiben in der Entwicklung der internen Genitalia können nicht als rachitische Symptome aufgefaßt werden, so daß es sehr fraglich bleibt, ob dieser Zwergwuchs rachitischer Ätiologie ist.

Es wäre doch wohl sehr merkwürdig, daß eine so schwere Rachitis keine Rumpf- und Extremitätendeformitäten und Asymmetrie hervorgerufen hätte, daß das ganze Skelett so grazil und kalkarm ist, währenddem ja doch meistens dicke auffallend harte und schwere Knochen nach der Heilung zurückbleiben.

A uch dieTatsache, daßdas Längenverhältnisderverschiedenen Extremitätenteile dasselbe ist, wie das ihrer erwachsenen Schwestern, spricht sehr gegen die rachitische Pathogenese.

Dort finden wir ja meist eine Störung in den Proportionen der einzelnen Abschnitte der Glieder, bei der die Unterextremitäten stärker als die Oberextremitäten, am meisten aber die Femora verkürzt sind (Schmidt), was in unserem Falle vollkommen fehlt.

Ubrigens ist uns auch in den Fällen, wo der Zwergwuchs auf die Rachitis zurückgeführt wird, das Wesen nicht bekannt. Bei der kolossalen Verbreitung der Rachitis, die nach Wiela nd bei $90 \%$ aller Kinder speziell der ärmeren Klassen, wahrgenommen wird, darf es sicher befremden, daß, wenn der rachitische Knochenprozeß als solcher $Z$ wergwuchs hervorrufen konnte, so wenig rachitische und viel weniger nicht deformierte rachitische $Z$ werge vorkommen.

Da ist es wohl wahrscheinlicher, eine tiefere Ursache zu vermuten, die vielleicht die Rachitis (?) selbst bedingt hat und unabhängig von dieser den Zwergwuchs. 
Ich glaube darum, in unserem Falle rachitischen Zwergwuchs ausschließen zu müssen.

Es gibt in unserem Falle ein anderes schädigendes Moment, das mit großer Wahrscheinlichkeit in Zusammenhang mit dem Zwergwuchs gebracht werden kann.

Unser Zwerg hat wahrscheinlich Lues hereditaria. Die Wassermannsche Reaktion des Blutes ist $6 / 10$ positiv. Diese Tatsache macht in Verbindung mit anamnestischen Daten und den anderen körperlichen Erscheinungen die Wahrscheinlichkeit fast zur Sicherheit.

Drei Monate alt, bekam das Kind eine schwere Erkrankung, wonach Idiotie und physische Unterentwicklung zurückblieb. Bis zu ihrem 10. Jahre hat die Patientin fortwährend Geschwüre auf ihrem Gesäß. Auf ihrer rechten Cornea eine Narbe. Die Extremitätenteile stehen so eigentümlich aufeinander, daß das Wort, gedreht" den allgemeinen Eindruck am besten wiedergibt. Die Schädelveränderungen gehören eher zur Lues wie zur Rachitis, besonders der von Parrot zuerst beschriebene natiforme Schädel soll nach Fournier für die hereditäre Lues pathognomisch sein.

Kann also die wohl vorhandene kongenitale Lues in unserem Falle als direkte Ursache des $Z$ wergwuchses und der anderen Erscheinungen aufgefaßt werden? Fournier ist überzeugt, daß mehrere Fälle von Nanisme vraie unzweifelhaft auf Heredolues beruhen und zitiert 5 Fälle aus der Literatur, wo nach seiner Meinung die Heredolues sicher eine Rolle gespielt hat.

Man könnte aber aus diesen Beschreibungen entnehmen, daß in der Mehrzahl dieser Fälle der proportionierte Zwergwuchs oder Zwergwuchs mit infantilen Proportionen die Regel war.

Von einer deutlichen Disharmonie oder Difformität war in keinem der Fälle die Rede.

Auch die Idiotie ist sehr häufig eine Folge der kongenitalen Lues. Daß in unserem Falle eine syphilitische Skelettaffektion die Ursache des Zwergwuchses ist, läßt sich schwer von der Hand weisen, aber dennoch gilt dasselbe, was wir bei der Besprechung der Rachitis gesagt haben. In den meisten Fällen ist eine syphilitische Skelettaffektion Ursache vermehrten Längenwachstums der Knochen. (S. v. d. Valk).

Wäre die kongenitale luetische Skelettaffektion wirklich die Ursache des Zwergwuchses, dann ist in Anbetracht der relativen Häufigkeit der kongenitalen Syphilis, die relative Seltenheit des Zwergwuchses auffallend.

In meinem Falle besteht wahrscheinlich eine andere durch die Syphilis hervorgerufene Störung, die als Ursache des Zwergwuchses aufgefaßt werden kann.

Es gibt nun Erscheinungen, die darauf hinweisen, daß andere Noxa hier im Spiele sind. 
Die ausgesprochene Obesitas, die infantilen Genitalia interna, die große Zunge, die trophischen Hautstörungen zeigen mit Deutlichkeit darauf hin. Auch die Kombination des Zwergwuchses mit Idiotie, die beide nach einer heftigen Staupe im dritten Lebensmonat entstanden sind, bringen mich dazu, die verschiedenen Störungen einheitlich zu betrachten.

Wie wir schon im Anfang kurz besprochen, steht Wachstum und Entwicklung unter dem Einfluß der Blutdrüsen. Klinik und Experiment haben den mächtigen Einfluß vieler dieser Organe, nicht nur auf die Entwicklung des Knochensystems, sondern des ganzen Individuums unwiderlegbar dargetan.

Ausfall der Schilddrüsenfunktion führt zu Zwergwuchs und eine Reihe von Erscheinungen, die wir bei der Thyreoaplasie haben kennen gelernt.

Es ist bekannt, daß dabei häufig hochgradige Idiotie auftritt. Aus den Mitteilungen verschiedener Untersucher geht hervor, daß die Arme und Beine im Verhältnis zum Rumpf oft zu klein sind. Es liegt also auf der Hand, wo es sich um einen idiotischen disproportionierten $Z_{w}$ erg handelt, an eine A- oder Hypofunktion der Schilddrüse zu denken, um so mehr, wo die große Zunge ein Charakteristicum der Thyreoaplasie bildet, um so mehr als die interessanten Beobachtungen meines früheren Lehrers Professor P. K. Pel, überzeugend auf den kausalen Zusammenhang zwischen Wachstumsstörungen infolge Blutdrüsenerkrankungen und hereditärer Lues hinweisen.

Pel hat namentlich zwei Kinder eines Syphilitikers beschrieben; das eine hatte kongen. Myxödem, das andere Akromegalie nebst Infantilismus. (Berl. Klin. Wochenschr. 1905, Nr. 44a.) Dennoch läßt sich hier ein Ausfall der Schilddrüsenfunktion mit an Sicherheit grenzender Wahrscheinlichkeit ausschließen.

In unserem Falle fehlen die myxödematösen Hautveränderungen vollkommen, die Nasenwurzel ist nicht eingesunken, die Nase nicht breit, die Augenlider nicht geschwollen, es fehlen die dicken Lippen, das Vollmondantlitz, die rauhe Stimme, der so häufige Nabelbruch. Der Haarwuchs ist in unserem Falle im Gegensatz zu der Thyreoaplasie und dem infantilen Myxödem üppig. Auch die starken sekundären Geschlechtskennzeichen sprechen gegen eine Hypothyreose. Am meisten beweisend sind die Röntgenogramme.

Die Epiphysenfugen sind geschlossen, die Knochenkerne alle vollkommen entwickelt, was sicher bei der Schilddrüsenhypofunktion nicht vorkommen würde in diesem Alter.

Gerade die Verzögerung des Knochenwachstums ist beim infantilen und kongenitalen Myxödem eine sehr große.

Auch die Form der Idiotie unseres Falles paßt nicht zu der Annahme einer Schilddrüsenstörung. 
Aus ihrer Gebärdensprache, ihren Modulationen, ihrem Affektleben geht zur Genüge hervor, daß sie konkrete Begriffe gebildet hat. Sie weiß sogar in sehr geschickter Weise bei ihrer fehlenden Sprache deutlich zu machen, was sie verlangt. Sie hat die ausgesprochene Neigung, zu simulieren und durch verschiedene Laute ihre Zufriedenheit oder ihre Unzufriedenheit zu markieren. Dies könnte ja alles auch bei einer mittelschweren Myxidiotin vorkommen.

Dennoch findet man hier meistens eine gewisse Trägheit und apathischen Torpor. Gerade das Gegenteil ist bei unserer Patientin der Fall. Sie ist fortwährend in Bewegung, produziert fortwährend allerhand Laute und gehört also zu den erregten Formen mit meist heiterer Stimmung.

Es macht oft den Eindruck, als wäre die große Zunge mit das Hindernis der Spracherlernung gewesen.

Thre Aufmerksamkeit ist lebhaft, obschon schwer zu fesseln. Große Geschehnisse behält sie ziemlich gut. Sie ist stets in leichter Unruhe, klatscht in die Hände und trampelt mit den Füßen, lacht, ziert sich und hat die Neigung, was sie sieht, nachzuahmen. Sie zeigt also eine Form der Idiotie, die der der Thyreoaplasie oder des infantilen Myxödems nahezu fremd ist.

Interessant ist auch die Mitteilung der Verwandten, daß sie gerade in den letzten zehn Jahren sich psychisch gebessert habe, daß sie bis zu ihrem 20. Jahre, nach der Beschreibung sicher zu den tieferstehenden Idioten gerechnet werden mußte.

Wie dem auch sei, daß der Schilddrüse in der Pathogenese keine nennenswerte Rolle zukommt, geht wohl aus obigem hervor.

Auf der Suche nach einer anderen Blutdrüse, die für die Erscheinungen unserer Patientin verantwortlich gemacht werden könnte, kommt die Hypophysis an erster Stelle.

Um so mehr weil unsere Patientin eine cerebrale Erkrankung durchgemacht hat.

Welcher Prozeß ist nun die Ursache aller Erscheinungen? Sie hat bei ihren schlaffen Gelenken Spasmus bei passiven Bewegungen, ja die Patellar- und Achillessehnenreflexe sind sehr lebhaft. Häufig läßt sich Fußklonus nachweisen. Der Fußsohlenreflex ist im Sinne des Babins kischen Reflexes aufzufassen. Der Gang ist schwerfällig, breitspurig und macht einen unbeholfenen spastischen Eindruck.

Die Annahme, daß die Idiotie auf einer schweren organischen cerebralen Störung beruht, ist äußerst wahrscheinlich.

Daß nun als direkte Folge des Krankheitsprozesses, oder als Folge der sekundären Störungen z. B. ein Hydrocephalus internus die Hypophysis in ihrer Funktion gestört hat, darf sicher in den Bereich der Möglichkeit gezogen werden. 
Die Annahme eines Hydrocephalus hat gewiß vieles für sich, weil die Störungen, welche auf eine Beeinträchtigung des Zentralnervensystems weisen, symmetrisch ausgebildet sind.

Die Hydrocephalie könnte unschwer auf die ererbte Syphilis zurückgeführt werden. Andererseits ist auch die Möglichkeit nicht von der Hand zu weisen, daß der luetische Krankheitsprozeß die Hypophysis ergriffen hat. Eine Meinung, welche in den Untersuchungen Si m monds eine kräftige Stütze finden kann.

Sim monds fand nämlich in vielen Fällen von kongenitaler Syphilis gummöse Veränderungen der Hypophysis, speziell im Vorderlappen.

Bei dieser Sachlage liegt es auf der Hand, an eine Beeinträchtigung der Hypophyse zu denken.

Die Hypophyse übt, das steht unzweideutig fest, einen kolossalen Einfluß auf das Knochenwachstum aus.

Exstirpationsexperimente haben bewiesen, daß Vorderlappenausfall bei jungen Tieren Zwergwuchs hervorruft.

Biedl sagt in Anbetreff dieser Frage ,so viel kann aber als sichergestellt gelten, daß der Wegfall eines größeren Anteiles des Hypophysenvorderlappens bei jugendlichen Tieren eine Gruppe von Folgeerscheinungen nach sich zieht. Als solche werden verzeichnet: Störungen im Wachstum, Hemmung der Geschlechtsreife und eine auffallende Veränderung im Stoffwechsel, welche zu einer erheblichen Zunahme des Körperfettes führt. Während man zunächst geneigt war, diesen Symptomenkomplex auf den Ausfall des Vorderlappens zu beziehen, kann heute mit Sicherheit eigentlich nur die Wachstumshemmung mit dem Fehlen des Vorderlappengewebes in Zusammenhang gebracht werden.

Bezüglich die Hypoplasie des Genitalapparates ist es auf Grund der vorliegenden Kenntnisse nicht zu entscheiden, ob sie ein Ausfallssymptom des Vorderlappens darstellt. Manches spricht dafür, daß ein partieller Funktionsausfall der Pars intermedia in erster Reihe in Betracht gezogen werden muß. Die Stoffwechselstörung ist zweifellos auf die letztere allein zu beziehen."

Auch die menschliche Pathologie gibt Beweise für den innigen $\mathrm{Zu}$ sammenhang zwischen Hypophysis und Knochenwachstum.

Hyperfunktion des Vorderlappens ist die Ursache der akromegalen Knochenveränderungen.

Auch der Riesenwuchs wird von manchen Autoren auf einen direkten, von anderen auf einen indirekten Einfluß des Hypophysisvorderlappens zurückgeführt.

Manche nehmen an, daß die A- und Hypofunktion der Hypophyse die Dystrophia adiposo-genitalis verursacht. Der sowohl bei dieser Krankheit wie bei Hypophysistumoren vorkommende Minder- resp. 
Zwergwuchs wird von den meisten Autoren auf eine Hypofunktion des Vorderlappens zurückgeführt.

Wie dem auch sei, welche Symptome auf einer Hypo-, welche auf einer Hyperfunktion beruhen, feststeht, daß bei Hypophysenerkrankungen Wachstumsstörungen, nämlich des Knochensystems, in zwei Richtungen vorkommen.

Wie wir uns die Wirkung dieses Organs vorstellen, sei hier nur mit einigen Worten angedeutet.

Die innige Korrelation der Blutdrüsen macht die Deutung eines bestimmten Symptoms als von einer Drüse abhängig fast unmöglich. Daß aber die Hypophysis einen anderen Einfluß auf das Knochenwachstum ausübt, wie z. B. Ovarien und Schilddrüse, geht mit Sicherheit daraus hervor, daß beim Adenom des Vorderlappens (Akromegalie) eine Vermehrung des Knochenwachstums auftritt, und zwar ein interstitielles (die Epiphysärscheiben sind geschlossen). Der Unterkiefer, der nicht durch Apposition und nur interstitiell wächst (Murk Jansen), wird größer. Dies sieht man bei keiner anderen Blutdrüsenaffektion.

Wird der Tumor weggenommen, dann wird der Kiefer wieder kleiner, und die Zähne nähern sich wieder. In den späteren Stadien der Akromegalie, wo vielleicht die Hypophyse ihre Funktion einstellt, trifft man eine Knochenatrophie.

Die Hypophysis scheint also hauptsächlich Einfluß zu üben auf die normalen $\mathrm{Ab}$ - und Zufuhr, auf die Apposition, Interposition und Resorption.

Die Hypophysis übt also einen Wachstumsreiz und nicht mehr aus. Tandler hat teilweise recht, wenn er behauptet, daß die Keimdrüsen das Maß des Wachstums bestimmen, die Hypophysis das Wachstum selbst bestimmt.

Wir dürfen aber den Einfluß der Schilddrüse auf das Knochensystem nicht vernachlässigen - müssen ihn aber als einen anderen auffassen, wie den der Hypophysis.

Afunktion der Schilddrüse hemmt die enchondrale und periostale Verknöcherung, regt diesen Prozeß bei Hyperfunktion an (Basedow in der Jugend, Holmgren). Die Zellteilung, die Verteilung der Baumaterialien steht unter ihrem Einfluß.

Die Hypophysis und Schilddrüse arbeiten zusammen, aber die erste hat vornehmlich mit dem Reiz zur Knochen- und Gewebeneubildung zu tun. Die Keimdrüsen greifen dann nach und nach ein durch eine Hemmung des ganzen Prozesses, und den Schluß der Epiphysärscheiben.

Schon Woods Hutchinson vermutete, daß der Hypopituitarismus Zwergwuchs verursachen könnte.

Er teilt einen Fall mit, wo die Obduktion einen Tumor des Hypophysenvorderlappens nachwies. In der deutschen Literatur wird dieser 
Fall stets als Chondrodystrophia ausgesprochen, was offensichtlich auf einen Irrtum zurückzuführen ist. Einige Seiten vor der Beschreibung dieses Zwerges steht das Photogramm eines chondrodystrophischen Skeletts. Beide sind aber vollkommen voneinander unabhängig (man lese das Original).

Bei Falta findet man eine große Zahl Fälle aus der Literatur, wo bei Hypophysenerkrankung Minderwuchs resp. Zwergwuchs bestand. In allen diesen Fällen waren auch die Genitalia infantil, und in den meisten Fällen bestand das Symptomenbild der Dystrophia adiposogenitalis.

Anatomisch wurden in vielen dieser Fälle Hypophysentumoren nachgewiesen.

Klinisch sind auch Fälle von Zwergwuchs mit Hypophysiserkrankungen in Zusammenhang gebracht worden (Aschner, Peritz).

Falta leugnet in den Fällen, wo keine Symptome der Dystrophia adiposo-genitalis bestehen, den Zusammenhang; nach meiner Meinung geht er darin zu weit.

Sicher, sekundäre Geschlechtscharaktere und Entwicklung der Genitalia interna gehen wohl meistens zusammen, und in weitaus der Mehrzahl der Hypophysistumoren verbindet sich damit ein Infantilbleiben des Individuums, was aber längst noch nicht beweist, daß bei einer A- oder Hypofunktion a usschließlich des Vorderlappens eine nachweisbare Störung der Genitalia vorhanden sein $m u ß$.

Die Art des Prozesses hat natürlich großen Einfluß, nicht der Tumor als solcher, sondern die Funktion des Organs ist der vornehmste Faktor.

Biedl sagt, daß bei 34 Fällen von Hypophysistumoren nur in 16 Fällen Genitalatrophie bestand, währenddem experimentell nur dies feststeht, daß die Wachstumsstörung ausschließlich auf den Vorderlappen zurückgeführt werden muß.

Der Fall Péritz mit normalen äußeren Genitalien wird auf Grund anderer Symptome mit Recht auf eine Störung der Funktion des Hypophysisvorderlappens zurückgeführt.

Es ist interessant, daß in den Fällen $\mathrm{Hu}$ utchinsons, Zuelers und $\mathrm{H}$ ueters Hypophysistumoren resp. Erkrankungen gefunden wurden ohne Infantilismus. Der Fall Bendas, Zwerg mit Hypophysistumor, der wohl infantil war, hatte in seiner Symptomatologie gar keine Ähnlichkeit mit der Dystrophia adiposo-genitalis.

Kehren wir jetzt zu unserem Fall zurück, wo Hirnerscheinungen mit Zwergwuchs bestehen.

Die Weygandtsche Auffassung, die gerade den Einfluß der Hypophysis bei Zwergwuchs mit Hirnerscheinungen betont, haben wir oben schon ausführlich mitgeteilt. 
Inwieweit Wachstumsstillstand nach schweren Schädeltraumen auf eine Funktionsstörung der Hypophysis zurückgeführt werden muB, können nur weitere Beobachtungen mit Sektionen lehren.

Unsere Patientin zeigt nun Erscheinungen, die auf eine Funktionsstörung der Hypophysis hinweisen, und zwar:

1. den Zwergwuchs und die kolossale Obesitas;

2. die albastfarbene trockene Haut, besonders an Hand- und Fußrücken ${ }^{1}$ );

3. das verspätete Auftreten der Menstruation, die infantile Entwicklung der Genitalia interna;

4. die Form der Idiotie, die sog. heitere Form, wie diese unter anderem durch Sprinzel in einem Falle von Hypophysistumor beobachtet wurde.

Alle diese Erscheinungen wären mit einem Hypopituitarismus in Verbindung zu bringen.

Andererseits zeigt unsere Patientin Erscheinungen, die mit unserer jetzigen Kenntnis über Hyper- und Hypofunktion sehr schwer als auf Hypofunktion beruhend aufgefaßt werden können, und zwar:

1. die Adrenalinglykosurie,

2. den akromegalen Kiefer und die große Zunge,

3. die kolossale Entwicklung der sekundären Geschlechtscharaktere.

Wir haben also zwei Gruppen von Erscheinungen, die auf gegensätzliche Funktionsstörungen hinweisen.

Man könnte geneigt sein anzunehmen, daß zwar der Hypophyse eine Bedeutung zukommt, daß aber, wenn wirklich Störungen der inneren Sekretion in Betracht kommen, mehrere Organe befallen sind.

Man muß aber mit dieser Auffassung vorsichtig sein, da gerade bei diesen Fällen von Hypophysisleiden in der Jugend eigentümliche Symptomgruppierungen, ja scharfe Kontraste in den Erscheinungen beschrieben worden sind.

Es gibt auch Fälle von Akromegalie in der Jugend, wo nur die Extremitäten größer werden, Zunge und Schädel aber nicht ( $\mathrm{Pel})$.

Auch Fälle, wo eine Hyperplasie der Genitalia bestand.

In dieser Hinsicht ist der Fall Schulzes und Fischers interessant, die eine Mischform bei einem elfjährigen Mädchen beschrieben (von Akromegalie und Dystrophia adiposo-genitalis).

1) Interessant sind die Mitteilungen Bertolottis (N. I. de la S. 1914, Nr. 1). Sie teilen einen Fall von Dystrophia adiposo-genitalis mit. Die Patientin war 39 Jahre alt und hatte eine Länge von 1,38 m. Die Extremitäten waren im mikromelen Sinne verkürzt, am meisten in den distalsten Abschnitten. Röntgenologisch wurde ein Teratom der Hypophyse diagnostiziert. Die Photogramme der Rückseite der Hand zeigen dieselbe eigenartige rauhe Oberfläche, wie wir sie bei unserer Patientin antreffen. 
Dennoch bleibt der Kontrast in den Erscheinungen unseres Falles schwer zu deuten.

Wir sehen also zahlreiche Hinweise auf den Zusammenhang der Erkrankungen mit der Hypophysis - bewiesen haben sie ihn nicht.

Auch die Röntgenphotogramme nicht.

Die heredo-luetische Ätiologie ist wohl nicht von der Hand zu weisen.

Ich habe etwas länger bei der Hypophysis verweilt in Hinsicht auf Weygands Betrachtungen und Biedls Ausspruch:

„Künftig müßte in allen Fällen von Zwergwuchs dem Verhalten der Hypophyse Beachtung geschenkt werden."

\section{Literaturverzeichnis.}

Für ausführliche Arbeiten, dieses Thema betreffend, verweise ich auf:

Hastings-Gilford, The disorders of post natal growth and development. London 1911.

Frangenheim, Die Krankheiten des Knochensystems im Kindesalter. Stuttgart 1913.

Wieland, Spezielle Pathologie des Bewegungsapparates im Kindesalter. Handbuch der allgem. Pathologie und der pathologischen Anatomie des Kindesalters. Bd. II. Abt. 1.

Gundobin, Die Erkrankungen der Blutdrüsen. Berlin 1913.

Biedl, Innere Sekretion. Bd. I u. II. Berlin u. Wien 1913.

Jansen (Murk), Achondroplasie, its nature and its cause. Leiden 1912. Das

Wesen und das Werden der Achondroplasie usw. Stuttgart 1913.

Fournier, Edw., Recherche et diagnostic de l'hérédo-syphilis. Paris 1907.

Weiter benutzte Literatur:

Aschner, Über einen Fall von hypophysärem Zwergwuchs mit Gravidität nebst Bemerkungen über die Ätiologie des Zwergwuchses. Zeitschr. f. Geburtsk. u. Gynäkol. 32, 644.1910.

Bertolotti, Polydactylie et teratome hypophysaire. Nouvelle Iconographie de la Salpêtrière 1914, Nr. 1.

Benda, Berl. klin. Wochenschr. 1900 (zit. aus Péritz).

Cestan, A propos d'un cas d'Achondroplasie. Nouvelle Iconographie de la Salpêtrière 14. 1901.

Evan (James), Some manifestations of pituitary growths. Brit. med. Journ., 2. Dez. 1911.

Gulecke, Zwergwuchs infolge prämaturer Synostose. Archiv f. klin. Chir. 88. 1907.

Hutchinson (Woods), The pituitary gland as a factor in acromegaly and gigantisme. New York med. Journ. 6r. 1898. 72. 1900.

Hueter, Hypophysistuberkulose bei einer Zwergin. Virchows Archiv 1905.

Holmgren, Über das Längenwachstum bei Hyperthyreose. Med. Klin. 1910.

Hanseman, Echte Nanosomie usw. Berl. klin. Wochenschr. 1902, Nr. 52.

Joachimsthal, Über den Zwergwuchs und verwandte Wachstumsstörungen.

Deutsche med. Wochenschr. 1899, Nr. 17 u. 18.

Jansen, Murk, Rachitis en Dwerggroei. Ned. Tijdschr. v. Geneesk. 1914, I, S. 864 . 
Levi, Ett., Contribution à la connaissance de la microsomie etc. Nouvelle Iconographie de la Salpêtrière 1910.

Pel, P. K., Akromegalie partielle avec infantilisme. Nouvelle Iconographie de la Salpetriere 19. 1906.

Pel, P. K., Familiäres Vorkommen von Akromegalie und Myxödem auf luetischer Grundlage. Berl. klin. Wochenschr. 1905, Nr. 44.

P éritz, Hypophysenerkrankungen. Monatsschr. f. Psych. u. Neurol. 33, 8404.

Simmonds, Ưber syphilitische Erkrankungen der Hypophysis, insbesondere bei Lues congenita. Dermatol. Wochenschr. 58, 1914, Ergänzungsheft.

Schrumpf, Über das klinische Bild der Achondroplasie beim Erwachsenen und eine ihr sehr ähnliche, bisher noch nicht bescbriebene Form von mikromelem Zwergwuchs bei einer 56jährigen Frau. Berl. klin. Wochenschr. 1908, Nr. 48, S. 2137.

Sterling, W., Ein Fall von rachitischem Zwergwuchs, kombiniert mit hysterodegenerativer Psychose. Zeitschr. f. d. ges. Neur. u. Psych. 19, Heft 3. 1913.

Sprinzels, Gesellschaft der Ärzte in Wien, 7. Juni. Wiener klin. Wochenschr. 1912, Nr. 24.

Schulze und Fischer, Zur Lehre von der Akromegalie und Osteoarthropathie hypertrophiante. Mitt. a. d. Grenzgeb. d. med. u. Chir. 24, 607. 1902.

Tandler, Über den Einfluß der innersekretorischen Anteile der Geschlechtsdrüsen auf die äußeren Erscheinungen des Menschen. Wiener klin. Wochenschr. 1910, Nr. 13.

Valk, W. v. d., Been en gewrichtslues. Ned. Tijdschr. v. Geneesk. 1909, I, 6, S. 2008.

Weygandt, Schwachsinn und Hirnkrankheiten mit Zwergwuchs. Monatsschr. f. Psych. u. Neurol. 35, Heft 1. 1914.

Zimmern, Sur un cas de rachitisme familial. Nouvelle Iconographie de la Salpêtrière 14. 1901.

Zöllner, Tumor der Schädelbasis, ausgehend von der Hypophyse. Archiv f. Psych. 44. 1908. 